\title{
Estimativa de ingestão de agrotóxicos organofosforados pelo consumo de frutas e hortaliças
}

\author{
Estimation of organophosphate pesticide ingestion \\ through intake of fruits and vegetables
}

\author{
Verona Borges Ferreira1,2, Thadia Turon Costa da Silva ${ }^{1,2,3}$, \\ Silvia Regina Magalhães Couto Garcia ${ }^{2,3}$, Armando Ubirajara Oliveira Sabaa Srur ${ }^{1,2,3}$
}

\begin{abstract}
Resumo
Introdução: A agricultura no Brasil caracteriza-se pelo uso elevado de agrotóxicos. Os inseticidas organofosforados estão entre a classe de agrotóxicos mais intensamente aplicados. Objetivo: Estimar a ingestão de resíduo de agrotóxicos organofosforados por crianças e adultos, considerando o consumo da população brasileira e a recomendação diária de frutas e hortaliças (FH). Método: Foram calculados a Estimativa Diária de Ingestão (EDI) e o percentual da Ingestão Diária Aceitável (\%IDA) de acordo com a metodologia proposta pela WHO e FAO, em que \%IDA > 100 caracteriza um possível risco de ingestão de agrotóxicos. Os dados sobre a concentração do agrotóxico no alimento foram obtidos do Programa de Análise de Resíduo de Agrotóxicos. Os dados sobre consumo do alimento e o peso corpóreo da população em estudo foram obtidos da Pesquisa de Orçamento Familiar de 2008-2009. A recomendação de ingestão de FH preconizada para uma dieta saudável foi de 400 gramas/dia. Resultados: Com base no consumo alimentar da população brasileira, apenas o agrotóxico metidationa excedeu o parâmetro toxicológico de segurança para crianças. Caso o consumo de $\mathrm{FH}$ pela população brasileira alcançasse a recomendação da WHO, seis agrotóxicos excederiam o \%IDA para crianças: diazinona, dicofol, dimetoato, metamidofós, metidationa e protiofós. Já para adultos, três agrotóxicos excederiam o \%IDA: dicofol, metamidofós e metidationa, deflagrando o uso indiscriminado desses inseticidas. Conclusão: A promoção do consumo de $\mathrm{FH}$ deve vir acompanhada de programas de monitoramento da comercialização, do uso desses agentes químicos e dos seus resíduos nos alimentos.
\end{abstract}

Palavras-chave: organofosforados; Limite Máximo de Resíduos (LMR); segurança alimentar.

\footnotetext{
Abstract

Background: The agriculture of Brazil is characterized by the high use of pesticides. Among the most intensely applied pesticides is the class of organophosphate insecticides. Objective: Estimate organophosphate pesticide ingestion in children and adults considering the consumption and daily recommended intake of fruits and vegetables (FV). Method: Estimated Daily Intake (EDI) and percentage of Acceptable Daily Intake (ADI) were calculated according to the methodology proposed by WHO and FAO, where $\% A D I>100$ features possible risk of pesticide ingestion. Data on pesticide residue in food obtained from the Pesticide Residue Analysis Program were used. National diet and body weight data of the studied population were obtained from the Household Budget Survey of 2008-2009. The recommended FV intake for a healthy diet used in this study was 400 grams/day. Results: Based on the Brazilian population's daily food intake, methidathion was the only pesticide to exceed the toxicology standard for

'Universidade Federal Rural do Rio de Janeiro (UFRRJ) - Seropédica (RJ), Brasil.

${ }^{2}$ Grupo de Pesquisa em Processamento de Frutas e Hortaliças (GPPFH/CNPq), Universidade Federal do Rio de Janeiro (UFRJ) - Rio de Janeiro (RJ), Brasil. ${ }^{3}$ Instituto de Nutrição Josué de Castro (INJC), Universidade Federal do Rio de Janeiro (UFRJ) - Rio de Janeiro (RJ), Brasil.

Trabalho realizado no Instituto de Nutrição Josué de Castro (INJC), Universidade Federal do Rio de Janeiro (UFRJ) - Rio de Janeiro (RJ), Brasil. Endereço para correspondência: Thadia Turon Costa da Silva - Instituto de Nutrição Josué de Castro (INJC), Centro de Ciências da Saúde, Universidade Federal do Rio de Janeiro (UFRJ), Avenida Carlos Chagas Filho, 373, Bloco J, $2^{\circ}$ andar - Cidade Universitária - CEP: 21941-590 - Rio de Janeiro (RJ), Brasil Email: thadia@uol.com.br

Fonte de financiamento: Fundação Carlos Chagas Filho de Amparo à Pesquisa do Estado do Rio de Janeiro. Conflito de interesses: nada a declarar.
} 
children. If the FV intake reached the level recommended by WHO, six pesticides would exceed the \%ADI for children: diazinon, dicofol, dimethoate, methamidophos, methidathion and prothiofos, and three pesticides would exceed the \%ADI for adults: dicofol, methamidophos, and methidathion, showing the excessive use of these chemicals. Conclusion: Encouragement of FV consumption must be accompanied by programs that monitor the commercialization and use of these pesticides, as well as their residues in food. Keywords: organophosphates; Maximum Residue Limit (MRL); food safety.

\section{INTRODUÇÃO}

No ano de 2009, o Brasil passou a ocupar a posição de maior consumidor mundial de agrotóxicos, ultrapassando a marca de 1 milhão de toneladas de substâncias tóxicas consumidas ${ }^{1}$. A produção de hortaliças no Brasil é caracterizada pelo consumo intensivo de agrotóxico e pelo não cumprimento das boas práticas agrícolas, uma vez que são encontrados limites superiores aos permitidos pela legislação nesses alimentos, além da presença de ingredientes ativos não permitidos para determinadas culturas ${ }^{2,3}$. Os inseticidas organofosforados estão entre a classe de agrotóxicos mais intensamente aplicados no Brasil, como o metamidofós e o acefato ${ }^{4}$.

Esses pesticidas fazem parte de uma categoria de agrotóxicos de elevada toxidade neurológica, pois atuam inibindo a enzima acetilcolinesterase, que é responsável por degradar a acetilcolina, impedindo que o estímulo nervoso seja cessado, acarretando um distúrbio chamado de crise colinérgica ${ }^{5}$. Vale ressaltar que a exposição prolongada a doses subclínicas desses compostos tóxicos a longo prazo também resulta em consequências neurológicas, com danos aos neurônios colinérgicos, como deficiências neuropsiquiátricas e neurocomportamentais persistentes, incluindo déficits de memória, cognitivo, mental, emocional, motor e sensorial ${ }^{6}$.

Tanto os organofosforados como os organoclorados apresentam elevada toxicidade aos seres humanos. No entanto, os organoclorados foram substituídos pelos inseticidas organofosforados, que, apesar dos efeitos colinérgicos, são menos persistentes no meio ambiente ${ }^{7}$.

Mesmo com os níveis elevados de resíduos de agrotóxicos nos alimentos brasileiros ${ }^{8}$, o consumo diário de frutas e hortaliças $(\mathrm{FH})$ deve ser estimulado devido à contribuição desses alimentos para a proteção da saúde e a diminuição do risco de ocorrência de doenças crônicas não transmissíveis, como obesidade, diabetes tipo 2, doenças cardiovasculares e certos tipos de câncer ${ }^{9,10}$. No entanto, menos de $10 \%$ da população brasileira atinge as recomendações de consumo de $\mathrm{FH}$, o qual é de, no mínimo, $400 \mathrm{~g}$ por dia para garantir de 9 a $12 \%$ da energia diária consumida, considerando uma dieta de $2 \mathrm{mil} \mathrm{Kcal} / \mathrm{dia}^{10,11}$. A Pesquisa Nacional de Saúde ${ }^{12}$ verificou um percentual de consumo mais elevado de $\mathrm{FH}$, em que 37,3\% da população acima de 18 anos alcançou as recomendações de consumo desses alimentos.

Em função do exposto, julga-se pertinente a estimativa da ingestão dietética de agrotóxicos, que se baseia na comparação da exposição humana com um parâmetro toxicologicamente seguro $^{13}$. Vale ressaltar que a Ingestão Diária Aceitável (IDA) é a quantidade máxima de substância que, ingerida diariamente durante toda a vida, parece não oferecer risco apreciável à saúde, de acordo com os conhecimentos atuais ${ }^{13}$. Embora seja um parâmetro para garantir a saúde da população exposta a alimentos com agrotóxicos, o cálculo da IDA não considera os efeitos da combinação de vários agrotóxicos ingeridos em uma mesma refeição ou ao longo do mesmo dia, o que pode se agravar à medida que esse agrotóxico é encontrado em vários alimentos consumidos na dieta cotidiana ${ }^{14}$. Dessa forma, o presente estudo estimou a ingestão de resíduo de agrotóxicos organofosforados a partir do consumo e da recomendação de consumo de frutas e hortaliças.

\section{MÉTODO}

A estimativa de ingestão de agrotóxicos organofosforados pelo consumo de frutas e hortaliças foi realizada de acordo com a metodologia proposta pela World Health Organization (WHO) e pela Food and Agricultura Organization of the United Nations (FAO) $)^{13,15}$, com o cálculo da Estimativa Diária de Ingestão (EDI) e do percentual da Ingestão Diária Aceitável (\%IDA), sendo necessários dados sobre a concentração do agrotóxico no alimento (Ri), o consumo do alimento (Ci) e o peso corpóreo (PC) da população em estudo (Equação 1 e 2). $\mathrm{O}$ risco pode existir quando a exposição ultrapassa o parâmetro toxicológico de segurança $\left(\% \mathrm{IDA}_{(\mathrm{EDI})}>100 \%\right)$.

$E D I=\Sigma R i x C i$

$\% I D A_{(E D I)}=\frac{E D I x 100}{I D A x P C}$

Os dados sobre a concentração de agrotóxico nos alimentos foram obtidos com base nos resultados do Programa de Análise de Resíduo de Agrotóxicos (PARA) da Agência Nacional de Vigilância Sanitária (ANVISA) ${ }^{3}$. Foram avaliados 16 ingredientes ativos da categoria dos organofosforados: acefato, clorpirifós, diazinona, diclorvós, dicofol, dimetoato, fenitrotiona, fentoato, fosmete, malationa, metamidofós, metidationa, parationa metílica, profenofós, protiofós e triazofós. Fizeram parte do estudo 16 frutas e hortaliças selecionadas pela ANVISA para serem monitoradas pelo PARA: abacaxi, alface, batata, beterraba, cebola, cenoura, couve, laranja, maçã, mamão, manga, morango, pepino, pimentão, repolho e tomate. Foi utilizado o resultado do PARA referente ao ano de 2010 no presente estudo, uma vez 
que o resultado dos anos consecutivos contempla um número menor de culturas selecionadas, o que poderia comprometer o resultado final do presente trabalho. A IDA foi utilizada como parâmetro toxicológico de segurança, e seus dados foram obtidos das Monografias dos Ingredientes Ativos ${ }^{16}$. Para os agrotóxicos com monografia excluída ou sem IDA no Brasil, foram utilizadas informações do Joint FAO/WHO Meeting on Pesticide Residues (JMPR) ${ }^{17}$.

A estimativa de ingestão de resíduo de agrotóxicos organofosforados em crianças foi realizada em função do consumo e da recomendação de frutas e hortaliças. Os dados referentes ao consumo foram obtidos da Pesquisa de Orçamento Familiar (POF) de 2008-2009 ${ }^{18}$, tendo como base o consumo alimentar médio per capita. A recomendação utilizada de frutas e hortaliças preconizada para uma dieta saudável foi a da $\mathrm{WHO}^{19}$, de 400 gramas/dia, sendo possível a comparação entre os riscos de ingestão de agrotóxico da dieta atual consumida pela população estudada e o potencial risco relacionado ao consumo preconizado para alcançar uma dieta adequada. O peso corpóreo utilizado foi de 33,85 Kg referente à média nacional para crianças de 10 anos e de $67,76 \mathrm{Kg}$ referente à média nacional para adultos, segundo dados da POF 2008-2009²0.

\section{RESULTADOS}

A estimativa de ingestão de resíduo de agrotóxicos organofosforados na dieta de crianças e de adultos, a partir do consumo e da recomendação de FH, está exposta na Tabela 1, que apresenta as EDI médias e os \% $\mathrm{IDA}_{(\mathrm{EDI})}$. A metidationa foi enquadrada em situação de risco à saúde humana para crianças por apresentar elevado \%IDA consumo e recomendação de $\mathrm{FH}$, respectivamente).

$\mathrm{Na}$ estimativa para o atendimento às recomendações de consumo de $400 \mathrm{~g} /$ dia de $\mathrm{FH}$, outros agrotóxicos, como metamidofós (366,6\%), diazinona (141,8\%), dicofol $(265,9 \%)$, dimetoato (100,3\%) e protiofós $(118,2 \%)$, também excederam o parâmetro toxicológico de segurança para crianças. Já para adultos, três agrotóxicos se enquadraram em situação de risco, apenas com base na estimativa para atender às recomendações de consumo de FH: dicofol (132,8\%), metamidofós (183,1\%) e metidationa (351,5\%). Com base no consumo de adultos, nenhum agrotóxico excedeu a IDA.

Nas Tabelas 2 e 3, os agrotóxicos estão distribuídos e

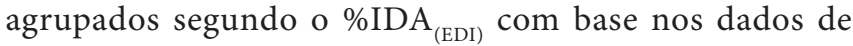
recomendação e consumo diário de $\mathrm{FH}$, tendo em vista o risco na dieta de crianças e de adultos, respectivamente. Dos avaliados em função da recomendação da $\mathrm{WHO}^{19}$, seis agrotóxicos excederam o parâmetro toxicológico de segurança para crianças: diazinona, dicofol, dimetoato, metamidofós, metidationa e protiofós. Para adultos, apenas três agrotóxicos excederam esse limite: dicofol, metamidofós e metidationa. Já com base no consumo alimentar médio per capita desses alimentos, apenas um agrotóxico, metidationa, excedeu esse parâmetro, e, somente quando se avaliou o risco para a dieta da criança, esses ingredientes ativos apresentaram \%IDA $>100$.

Tabela 1. Comparação entre Ingestão Diária Aceitável (IDA), Estimativa Diária de Ingestão (EDI) média e \%IDA(EDI) de compostos organofosforados, segundo o consumo diário brasileiro per capita e a recomendação diária de consumo de frutas e hortaliças $(\mathrm{FH})$

\begin{tabular}{|c|c|c|c|c|c|c|c|}
\hline \multirow{3}{*}{$\begin{array}{l}\text { Ingrediente } \\
\text { ativo }\end{array}$} & \multirow{3}{*}{$\begin{array}{c}\text { IDA } \\
\text { (mg/dia) }\end{array}$} & \multicolumn{2}{|c|}{ EDI médio } & \multicolumn{4}{|c|}{$\%_{\text {IDA }}$} \\
\hline & & \multirow[t]{2}{*}{$\begin{array}{l}\text { Segundo consumo } \\
\text { diário brasileiro } \\
\text { per capita de FH } \\
(100 \mathrm{~g} / \mathrm{dia})\end{array}$} & \multirow[t]{2}{*}{$\begin{array}{c}\text { Segundo } \\
\text { recomendação } \\
\text { diária de consumo } \\
\text { de FH }(400 \mathrm{~g} / \mathrm{dia})\end{array}$} & \multicolumn{2}{|c|}{$\begin{array}{c}\text { Segundo } \\
\text { consumo diário } \\
\text { brasileiro per capita de } \\
\text { FH }(100 \mathrm{~g} / \mathrm{dia})\end{array}$} & \multicolumn{2}{|c|}{$\begin{array}{c}\text { Segundo recomendação } \\
\text { diária de } \\
\text { consumo de FH } \\
(400 \mathrm{~g} / \mathrm{dia})\end{array}$} \\
\hline & & & & Criança & Adulto & Criança & Adulto \\
\hline Acefato & 0,03 & 0,030 & 0,120 & 3,0 & 1,5 & 12,2 & 6,1 \\
\hline Clorpirifós & 0,01 & 0,020 & 0,070 & 5,3 & 2,7 & 21,3 & 10,6 \\
\hline Diazinona & 0,002 & 0,020 & 0,100 & 35,4 & 17,7 & 141,8 & 70,8 \\
\hline Diclorvós & 0,004 & 0,010 & 0,050 & 8,6 & 4,3 & 34,5 & 17,2 \\
\hline Dicofol & 0,002 & 0,050 & 0,180 & 66,5 & 33,2 & 265,9 & 132,8 \\
\hline Dimetoato & 0,002 & 0,020 & 0,070 & 25,1 & 12,5 & 100,3 & 50,1 \\
\hline Fenitrotiona & 0,005 & 0,003 & 0,010 & 1,7 & 0,86 & 6,8 & 3,4 \\
\hline Fentoato & 0,003 & 0,002 & 0,010 & 1,5 & 0,74 & 5,9 & 2,9 \\
\hline Fosmete & 0,005 & 0,010 & 0,040 & 6,2 & 3,1 & 24,8 & 12,4 \\
\hline Malationa & 0,3 & 0,004 & 0,020 & 0,04 & 0,02 & 0,2 & 0,1 \\
\hline Metamidofós & 0,004 & 0,120 & 0,500 & 91,6 & 45,8 & 366,6 & 183,1 \\
\hline Metidationa & 0,001 & 0,060 & 0,240 & 175,9 & 87,9 & 703,7 & 351,5 \\
\hline Parationa metílica & 0,003 & 0,004 & 0,020 & 4,0 & 1,9 & 15,9 & 7,9 \\
\hline Profenofós & 0,01 & 0,070 & 0,290 & 21,6 & 10,8 & 86,4 & 43,1 \\
\hline Protiofós & 0,0001 & 0,001 & 0,004 & 29,5 & 14,8 & 118,2 & 59,0 \\
\hline Triazofós & 0,001 & 0,010 & 0,030 & 19,5 & 9,7 & 78 & 39,0 \\
\hline
\end{tabular}


Tabela 2. Distribuição dos ingredientes ativos segundo o \%IDA(EDI) com base na recomendação de consumo diário de frutas e hortaliças (FH) e no consumo diário brasileiro per capita de $\mathrm{FH}$ para crianças

Ingredientes ativos

\begin{tabular}{|c|c|c|}
\hline$\%_{\text {IDA }}$ & $\begin{array}{c}\text { Segundo } \\
\text { recomendação }{ }^{19}\end{array}$ & Segundo consumo ${ }^{18}$ \\
\hline $0-25 \%$ & $\begin{array}{l}\text { Acefato, clorpirifós, } \\
\text { fenitrotiona } \\
\text { fentoato, fosmete, } \\
\text { malationa e parationa } \\
\text { metílica }\end{array}$ & $\begin{array}{l}\text { Acefato, clorpirifós, } \\
\text { diclorvós } \\
\text { fenitrotiona, fentoato, } \\
\text { fosmete, malationa, } \\
\text { parationa metílica, } \\
\text { profenofós e triazofós }\end{array}$ \\
\hline$>25-50 \%$ & Diclorvós & $\begin{array}{l}\text { Diazinona, dimetoato e } \\
\text { protiofós }\end{array}$ \\
\hline $50-75 \%$ & - & Dicofol \\
\hline$>75-100 \%$ & Profenofós e triazofós & Metamidofós* \\
\hline$>100 \%$ & $\begin{array}{l}\text { Diazinona, dicofol, } \\
\text { dimetoato, } \\
\text { metamidofós*, } \\
\text { metidationa e protiofós }\end{array}$ & Metidationa \\
\hline
\end{tabular}

*Princípio ativo com monografia excluída, uso não permitido no País

Tabela 3. Distribuição dos ingredientes ativos segundo o \%IDA(EDI) com base na recomendação de consumo diário de frutas e hortaliças (FH) e no consumo diário brasileiro per capita de FH para adultos

\begin{tabular}{|c|c|c|}
\hline \multirow[b]{2}{*}{$\%_{\text {IDDI) }}$} & \multicolumn{2}{|c|}{ Ingredientes ativos } \\
\hline & $\begin{array}{c}\text { Segundo } \\
\text { recomendação }\end{array}$ & Segundo consumo ${ }^{18}$ \\
\hline $0-25 \%$ & $\begin{array}{l}\text { Acefato, clorpirifós, } \\
\text { diclorvós, fenitrotiona, } \\
\text { fentoato, fosmete, } \\
\text { malationa e parationa } \\
\text { metílica }\end{array}$ & $\begin{array}{l}\text { Acefato, clorpirifós, } \\
\text { diazinona, diclorvós, } \\
\text { dimetoato, fenitrotiona, } \\
\text { fentoato, fosmete, } \\
\text { malationa, parationa } \\
\text { metílica, profenofós, } \\
\text { protiofós e triazofós }\end{array}$ \\
\hline$>25-50 \%$ & Profenofós e triazofós & Dicofol e metamidofós \\
\hline$>50-75 \%$ & $\begin{array}{l}\text { Diazinona, dimetoato e } \\
\text { protiofós }\end{array}$ & - \\
\hline$>75-100 \%$ & - & Metidationa \\
\hline$>100 \%$ & $\begin{array}{l}\text { Dicofol, metamidofós }{ }^{*} \text { e } \\
\text { metidationa }\end{array}$ & - \\
\hline
\end{tabular}

*Princípio ativo com monografia excluída, uso não permitido no País

Sabe-se que o consumo de FH pela população brasileira, independente da faixa de renda, ainda está aquém das recomendações para uma dieta saudável, sendo necessário um aumento de pelo menos três vezes o consumo médio atual de $\mathrm{FH}$ da população ${ }^{11}$.

\section{DISCUSSÃO}

$\mathrm{A} \mathrm{FAO}^{13}$ classifica os agrotóxicos em quatro diferentes classes toxicológicas em função dos efeitos à saúde, decorrentes da exposição humana a esses agentes: classe I, ou extremamente tóxico; classe II, ou altamente tóxico; classe III, ou medianamente tóxico; e classe IV, ou pouco tóxico. Dos agrotóxicos que excederam o parâmetro de segurança, o metamidofós se enquadra na classe toxicológica
I, por ser considerado extremamente tóxico, enquanto o restante é classificado como classe II, altamente tóxicos ${ }^{13}$.

O metamidofós é um agrotóxico da classe dos organofosforados que está entre os três inseticidas mais consumidos no Brasil, assim como acefato e endosulfan, correspondendo, respectivamente, a 15,1, 11,2 e 7,3\% do consumo de inseticidas ${ }^{4}$. Esse agrotóxico estava em reavaliação pela ANVISA por apresentar toxicidade aguda e alto grau de neurotoxicidade e, em dezembro de 2012, teve sua monografia excluída e mantida exclusivamente para fins de monitoramento de resíduos ${ }^{4}$. No entanto, no atual estudo, ultrapassou o parâmetro de ingestão de segurança, caracterizando um risco à saúde na parcela da população estudada que consome FH nas quantidades recomendadas para uma dieta saudável.

Vale ressaltar que os agrotóxicos da classe dos organofosforados são considerados de elevada toxidade neurológica, pois possuem ação tóxica específica a nível simpático, inibindo a atividade da enzima acetilcolinesterase, que é responsável por hidrolisar acetilcolina, um neurotransmissor que se acumula como resultado dessa inibição, promovendo estimulação excessiva dos receptores muscarínicos, o que resulta em síndromes oculares, broncopulmonares, cardiovasculares e gastrointestinais com sintomas diversos ${ }^{21}$.

Esses efeitos tóxicos causados pelos inseticidas organofosforados são mais proeminentes em crianças. A exposição de neurotóxicos, embora demonstre não causar danos severos para adultos, pode resultar em danos permanentes se ocorrer durante o desenvolvimento pré-natal ou infância, caracterizados como períodos biológicos de intenso desenvolvimento neurológico ${ }^{22}$. Além disso, os organofosforados apresentam-se como disruptores endócrinos, podendo causar danos ao sistema reprodutor e desenvolvimento, especialmente para o sexo feminino. IñigoNuñez et al..$^{23}$ apontam quatro ingredientes ativos considerados agrotóxicos estrogênicos, clorpirifós, diazinona, cipermetrina e tiabendazol, dos quais os dois primeiros correspondentes à classe dos organofosforados. Pode-se ressaltar que, no presente estudo, a diazinona foi um dos agrotóxicos que excedeu o parâmetro toxicológico de segurança para crianças com base nos dados de recomendação da $\mathrm{WHO}^{19}$ para o consumo adequado de $\mathrm{FH}$.

O risco de ingestão dietética de agrotóxico está relacionado à contaminação do alimento, assim como a quantidade consumida do alimento pela população estudada. O presente trabalho apresenta os resultados com base no consumo alimentar médio per capita nacional, sem levar em consideração as características regionais, o que pode levar a diferentes resultados, uma vez que o consumo de FH pode diferir de uma região para a outra. Segundo dados da Pesquisa Nacional de Saúde ${ }^{12}$, o percentual de pessoas de 18 anos ou mais de idade que consumiam o equivalente a cinco porções diárias de FH foi de 28,2\% na Região Nordeste, 36,6\% no Norte, $42,8 \%$ no Sudeste, $34,2 \%$ no Sul e $43,8 \%$ no Centro-Oeste. Além disso, o mesmo estudo apresentou uma tendência de maior consumo com o aumento da idade e com o grau de escolaridade. 
$\mathrm{Na}$ Espanha, uma pesquisa que avaliou a estimativa de ingestão de agrotóxicos a partir do consumo de frutas observou percentuais da IDA inferiores aos apresentados no presente estudo. No entanto, os agrotóxicos organofosforados tiveram as maiores EDI. A diazinona apresentou a maior EDI, 13\% da IDA, comparada aos 35,4\% de IDA nas FH brasileiras ${ }^{23}$.

Estudo realizado por Nougadère et al. $^{17}$, na França, avaliou o risco crônico, considerando o consumo de resíduos de agrotóxico em alimentos desse país. Quanto à exposição média, 1,8\% dos ingredientes ativos estudados apresentou uma EDI superior à IDA: aldrin/dieldrin, heptacloro e mirex, considerados poluentes orgânicos persistentes, além do carbofurano, iodofenfós e dimetoato, classificados como inseticidas.

Em estudo realizado com 724 amostras de 46 diferentes tipos de FH em países da América Latina, incluindo o Brasil, foi encontrado resíduo acima do limite permitido de agrotóxico em $8,4 \%$ das amostras. O clorpirifós se destacou como um dos três ingredientes mais encontrados nas amostras analisadas ${ }^{24}$, o que não corrobora os resultados encontrados no presente trabalho, no qual clorpirifós não ultrapassou o limite toxicológico de segurança, apresentando como maior valor $21,3 \% \mathrm{IDA}_{(\mathrm{EDI})}$ apenas quando levado em consideração a recomendação de consumo de FH para crianças.

Em outro estudo realizado no Brasil sobre a exposição de pesticidas na dieta de estudantes universitários, o metamidofós foi o ingrediente ativo mais presente nas amostras estudadas, correspondendo a 37,7\% do total de amostras analisadas ${ }^{25}$.

A exposição cumulativa, que é a exposição simultânea a várias substâncias químicas na dieta, tem sido objeto de preocupação de órgãos reguladores, pois o efeito tóxico dessas substâncias pode ser aditivo, isto é, quando mais de um composto exerce o mesmo mecanismo de ação tóxica ${ }^{26}$. No caso em tela, seis agrotóxicos organofosforados presentes em FH excederam a IDA, sem levar em conta outros alimentos que compõem a dieta do brasileiro e que também são passíveis de contaminação.

Reiss et al. ${ }^{27}$ apresentaram estimativas do número potencial de casos anuais de câncer que poderiam ser prevenidos se metade da população dos Estados Unidos aumentasse seu consumo de FH em uma porção por dia, a partir de metanálise publicada de estudos epidemiológicos nutricionais. As estimativas resultantes são de que aproximadamente 20 mil casos de câncer por ano poderiam ser evitados com o aumento do consumo de $\mathrm{FH}$. Esse número é comparado com uma estimativa concomitante de casos de câncer que poderiam ser atribuídos à ingestão de resíduos de agrotóxico a partir do aumento da mesma porção de FH no consumo, pois são estimados 10 casos por ano de câncer em função do consumo adicional de agrotóxicos nas FH. Os autores concluem que, diante da magnitude da diferença entre o benefício e o risco, apesar da incerteza, os efeitos positivos para a saúde no aumento do consumo de frutas e hortaliças excedem qualquer efeito negativo associado com o aumento da ingestão de resíduos de agrotóxicos nesses alimentos.

Foi possível perceber que as FH consumidas no Brasil contêm níveis elevados de resíduos de agrotóxicos que podem representar uma preocupação do ponto de vista de saúde pública, dependendo da quantidade consumida e do peso corpóreo da população. Estudos futuros considerando as diferenças regionais de consumo dos alimentos, as diferentes faixas etárias e o grau de escolaridade podem contribuir para a estimativa de ingestão de agrotóxicos na população brasileira e o monitoramento do risco.

É dever do governo e do setor produtivo monitorar a comercialização e o uso de agentes químicos, como os agrotóxicos, e apoiar estudos que avaliem esses riscos, principalmente na população infantil, período de intenso desenvolvimento neurológico. No entanto, a promoção e o aumento no consumo de FH devem ser encorajados e fazer parte das políticas de saúde e segurança alimentar.

\section{REFERÊNCIAS}

1. Instituto Brasileiro do Meio Ambiente e dos Recursos Naturais Renováveis. Boletim anual de produção, importação, exportação e vendas de agrotóxicos no Brasil [Internet]. Brasília; 2013 [citado em 2014 mar 15]. Disponível em: http://ibama.gov.br/areas-tematicas-qa/relatorios-de-comercializacaode-agrotoxicos/pagina-3

2. Preza DLC, Nogueira TF, Augusto LGS. Práticas na comercialização e na indicação de agrotóxicos em região produtora de hortaliças no Estado da Bahia. Magistra. 2011;23(4):168-74.

3. Brasil. Agência Nacional de Vigilância Sanitária. Gerência Geral de Toxicologia. Programa de Análise de Resíduos de Agrotóxicos em alimentos (PARA): relatório de atividades de 2009. Brasília; 2010.

4. Instituto Brasileiro de Geografia e Estatística. Diretoria de Geociências. Indicadores de desenvolvimento sustentável. Rio de Janeiro; 2012.350 p. (Estudos e Pesquisas, Informação Geográfica; 9).
5. Moreira JC, Jacob SC, Peres F, Lima JS, Meyer A, Oliveira-Silva JJ, et al. Avaliação integrada do impacto do uso de agrotóxicos sobre a saúde humana em uma comunidade agrícola de Nova Friburgo, RJ. Cien Saude Colet. 2002;7(2):299-311. http://dx.doi.org/10.1590/S1413-81232002000200010.

6. Androutsopoulos VP, Hernandez AF, Liesivuori J, Tsatsakis AM. A mechanistic overview of health associated effects of low levels of organochlorine and organophosphorous pesticides. Toxicology. 2013;307:89-94. http://dx.doi. org/10.1016/j.tox.2012.09.011. PMid:23041710.

7. Baird C, Cann M. Química ambiental. 4. ed. Porto Alegre: Bookman; 2011. 844 p.

8. Jardim ANO, Caldas ED. Exposição humana a substâncias químicas potencialmente tóxicas na dieta e os riscos para saúde. Quim Nova. 2009;32(7):1898-909. http://dx.doi.org/10.1590/S0100-40422009000700036. 
9. World Health Organization. Resolution WHO 57.17: global strategy on diet, physical activity and health. In: Proceedings of the 57th World Health Assembly; 2004 may 22; Geneva. Geneva; 2004.

10. Brasil. Ministério da Saúde. Secretaria de Atenção à Saúde. CoordenaçãoGeral da Política de Alimentação e Nutrição. Guia alimentar para a população brasileira: promovendo a alimentação saudável. Brasília; 2005. 236 p. (Série A, Normas e Manuais Técnicos).

11. Instituto Brasileiro de Geografia e Estatística. Coordenação de Trabalho e Rendimento. Pesquisa de orçamentos familiares 2008-2009: avaliação nutricional da disponibilidade domiciliar de alimentos no Brasil. Rio de Janeiro; 2010. 54 p.

12. Instituto Brasileiro de Geografia e Estatística. Diretoria de Pesquisas. Pesquisa Nacional de Saúde 2013: percepção do estado de saúde, estilos de vida e doenças crônicas. Rio de Janeiro; 2014. 181 p.

13. Food and Agricultural Organization of the United Nations. Submission and evaluation of pesticide residues data for the estimation of maximum residue levels in food and feed. 2nd ed. Rome; 2009. (FAO Plant, Production and Protection Paper; 197).

14. Carneiro FF, Pignati W, Rigotto RM, Augusto LGS, Rizollo A, Muller NM, et al. Dossiê ABRASCO: um alerta sobre os impactos dos agrotóxicos na saúde. $1^{\text {a }}$ parte. Rio de Janeiro: ABRASCO; 2012. 98 p.

15. World Health Organization. Global Environment Monitoring System/ Codex Alimentarius Commission Guidelines for predicting dietary intake of pesticides residues. Geneva; 1997.

16. Agência Nacional de Vigilância Sanitária. Monografias de agrotóxicos [Internet]. Brasília; 2012 [citado em 2012 mar 20]. Disponível em: http:// portal.anvisa.gov.br/wps/portal/anvisa/home/agrotoxicotoxicologia/ monografiasdeagrotóxicos

17. Nougadère A, Reninger JC, Volatier JL, Leblanc JC. Chronic dietary risk characterization for pesticide residues: a ranking and scoring method integrating agricultural uses and food contamination data. Food Chem Toxicol. 2011;49(7):1484-510. http://dx.doi.org/10.1016/j.fct.2011.03.024. PMid:21421018.

18. Instituto Brasileiro de Geografia e Estatística. Coordenação de Trabalho e Rendimento. Pesquisa de orçamentos familiares 2008-2009: análise do consumo alimentar pessoal no Brasil. Rio de Janeiro; 2011. 150 p.
19. World Health Organization. Expert Consultation. Diet, nutrition and the prevention of chronic diseases. Geneva; 2003. (Technical Report Series; 916).

20. Instituto Brasileiro de Geografia e Estatística. Coordenação de Trabalho e Rendimento. Pesquisa de orçamentos familiares 2008-2009: Antropometria e estado nutricional de crianças, adolescentes e adultos no Brasil. Rio de Janeiro; 2010. 130 p.

21. Fernández DG, Mancipe LC, Fernández DC. Intoxicación por organofosforados. Rev Fac Med. 2010;18(1):84-92. http://dx.doi.org/10.18359/rmed.1295.

22. Wiles R, Davies K, Campbell C. Overexposed: organophosphate insecticides in children's food. Washington: Environmental Working Group; 1998.

23. Iñigo-nuñez S, Herreros MA, Encinas T, Gonzalez-bulnes A. Estimated daily intake of pesticides and xenoestrogenic exposure by fruit consumption in the female population from a Mediterranean country (Spain). Food Control. 2010;21(1):471-7. http://dx.doi.org/10.1016/j.foodcont.2009.07.009.

24. Hjorth K, Johansen K, Holen B, Andersson A, Christensen HB, Siivinen $\mathrm{K}$, et al. Pesticide residues in fruits and vegetables from South America: a Nordic project. Food Control. 2011;22(11):1701-6. http://dx.doi.org/10.1016/j. foodcont.2010.05.017.

25. Caldas ED, Souza MV, Jardim ANO. Dietary risk assessment of organophosphorus and dithiocarbamate pesticides in a total diet study at a Brazilian university restaurant. Food Addit Contam. 2011;28(1):71-9. http://dx.doi.org/10.1080/19440049.2010.538935. PMid:21240829.

26. Jardim ANO, Caldas ED. Exposição humana a substâncias químicas potencialmente tóxicas na dieta e os riscos para saúde. Quim Nova. 2009;32(7):1898-909. http://dx.doi.org/10.1590/S0100-40422009000700036.

27. Reiss R, Johnston J, Tucker K, DeSesso JM, Keen CL. Estimation of câncer risks and benefits associated with a potential increased consumption of fruits and vegetables. Food Chem Toxicol. 2012;50(12):4421-7. http:// dx.doi.org/10.1016/j.fct.2012.08.055. PMid:22981907.

Recebido em: Jun. 16, 2015 Aprovado em: Set. 15, 2015 